\title{
Exact solutions of two-band models of graded-gap superlattices
}

\author{
B. Méndez and F. Domínguez-Adame \\ Departamento de Física de Materiales, Universidad Complutense, 28040 Madrid, Spain
}

\begin{abstract}
We have theoretically investigated two-band models of graded-gap superlattices within the envelope-function approximation. Assuming that the gap varies linearly with spatial coordinate, we are able to find exact solutions of the corresponding Dirac-like equation describing the conduction- and valenceband envelope-functions. The dispersion relation inside allowed miniband of the superlattice may be expressed in terms of confluent hypergeometric functions in a closed form.
\end{abstract}

PACS numbers: 73.20.Dx; 71.25.Cx; 73.61.Ey;

Typeset using REVTEX 
During the last years, graded-gap superlattices have been the subject of very detailed investigations as interesting materials for device applications [1,2]. The graded doping creates a modulation of both conduction- and valence-bands, which may be approximated by a sawtooth potential. The miniband structure can be obtained within the envelope-function approximation [3], the system being usually described by a scalar Hamiltonian (Schrödingerlike) corresponding to decoupled bands. However, this approach cannot adequately describe those graded-gap superlattices whose band modulation is comparable to the magnitude of the gap, and a more realistic band structure is essential to properly describe the electronic structure. In this paper we calculate the miniband structure of graded-gap superlattices within a two-band model, which is known to be valid in a large variety of semiconductor superlattices where the coupling of bands is not negligible, as occurs in some narrow-gap III-V compounds (InAs, InSb, GaSb).

We obtain the miniband structure in the superlattice by means of the effective-mass $\mathbf{k} \cdot \mathbf{p}$ approximation. There are two coupled envelope-functions describing the conductionband and valence-band states of the semiconductor, subject to an effective $2 \times 2$ Dirac-like equation along the growth direction $z$

$$
\left(-i \hbar v \sigma_{x} \partial+\frac{1}{2} E_{g}(z) \sigma_{z}-E\right)\left(\begin{array}{c}
f_{c}(z) \\
f_{v}(z)
\end{array}\right)=0,
$$

where $\partial=d / d z, \sigma_{x}$ and $\sigma_{z}$ are Pauli matrices, and $E_{g}(z)$ stands for the position-dependent gap in the two-band semiconductor superlattice. The spatial periodicity of the lattice implies that $E_{g}(z+L)=E_{g}(z), L$ being the period of the superlattice. We assume that the centre of the gap remains unchanged when doping; this simplifies calculations and is a good approximation in several cases (for instance, in $\mathrm{GaAs}_{-} \mathrm{Ga}_{1-x} \mathrm{Al}_{x} \mathrm{As}$ the centre of the gap varies only $10 \%$ of the gap difference in both materials). The velocity $v=\left(E_{g} / 2 m^{*}\right)^{1 / 2}$ is almost constant in direct gap III-V semiconductors, and we will assume this constancy hereafter. In graded-gap structures the gap varies linearly with position so that we can write

$$
E_{g}(z)=E_{g 0}+\left(E_{g L}-E_{g 0}\right)\left(\frac{z}{L}\right) \equiv E_{g 0}+\Delta E_{g}\left(\frac{z}{L}\right)
$$


where $E_{g 0}=E_{g}(0)$ and $E_{g L}=E_{g}(L)$ for $0<z<L$. Note that $E_{g}(z)$ is equivalent to a relativistic scalar-like potential in the Dirac theory, that is to say, Eq. (1) is analogous to the Dirac equation for a relativistic particle with a position-dependent mass. We exploit this analogy to find the exact solutions of Eq. (四) for the graded-gap (目). It is well-known that the Dirac equation for linear scalar-like potentials admits exact solutions (see Refs. [4.5] and references therein) and we can use a similar method to solve Eq. (11). Therefore we define

$$
\left(\begin{array}{c}
f_{c}(z) \\
f_{v}(z)
\end{array}\right)=\left(-i \hbar v \sigma_{x} \partial+\frac{1}{2} E_{g}(z) \sigma_{z}+E\right)\left(\begin{array}{c}
i \phi(z) \\
\phi(z)
\end{array}\right),
$$

and inserting (3) in (11) one obtains the equation for the function $\phi(z)$ as

$$
\left[-\hbar^{2} v^{2} \partial^{2}+\frac{1}{4} E_{g}^{2}(z)-E^{2}-\left(\frac{\hbar v \Delta E_{g}}{2 L}\right)\right] \phi(z)=0 .
$$

The equation (14) may be reduced to a standard form, the equation of the parabolic cylinder, by making the change of parameters according to

$$
\begin{aligned}
& x=\sqrt{\frac{L}{\hbar v \Delta E_{g}}}\left(E_{g 0}+\Delta E_{g} \frac{z}{L}\right), \\
& \eta=\frac{E^{2} L}{\hbar v \Delta E_{g}} .
\end{aligned}
$$

On making these substitutions one gets

$$
\frac{d^{2} \phi(x)}{d x^{2}}+\left(-\frac{x^{2}}{4}+\eta+\frac{1}{2}\right) \phi(x)=0
$$

whose two independent solutions are parabolic cylinder functions $D_{\eta}(x)$ and $D_{\eta}(-x)$. Using Eq. (3) we find that the envelope-functions in the conduction- and valence-bands can be cast in the matrix form

$$
\left(\begin{array}{c}
f_{c}(z) \\
f_{v}(z)
\end{array}\right)=\mathbf{D}[x(z)]\left(\begin{array}{c}
A \\
B
\end{array}\right), \quad 0<z<L,
$$

where $A$ and $B$ are arbitrary constants and the $2 \times 2$ matrix $\mathbf{D}[x(z)]$ is written out explicitly as 


$$
\mathbf{D}[x(z)]=\left[\begin{array}{cc}
-i\left\{D_{\eta}(x)-\sqrt{\eta} D_{\eta-1}(x)\right\} & -i\left\{D_{\eta}(-x)+\sqrt{\eta} D_{\eta-1}(-x)\right\} \\
D_{\eta}(x)+\sqrt{\eta} D_{\eta-1}(x) & D_{\eta}(-x)-\sqrt{\eta} D_{\eta-1}(-x)
\end{array}\right] .
$$

Once the general solution of the Dirac equation (1) is obtained, appropriate boundary conditions should be used to find eigenenergies. We assume the continuity of the envelopefunctions at the interface $z=L$, namely,

$$
\left(\begin{array}{c}
f_{c}\left(L^{-}\right) \\
f_{v}\left(L^{-}\right)
\end{array}\right)=\left(\begin{array}{c}
f_{c}\left(L^{+}\right) \\
f_{v}\left(L^{+}\right)
\end{array}\right)
$$

along with the Bloch condition in the growth direction

$$
\left(\begin{array}{c}
f_{c}(L) \\
f_{v}(L)
\end{array}\right)=\exp (i k L)\left(\begin{array}{c}
f_{c}(0) \\
f_{v}(0)
\end{array}\right)
$$

where $k$ denotes the component of the momentum along the growth direction $z$. By means of the general solution (7) we can find the dispersion relation as

$$
\cos k L=\frac{1}{2} \operatorname{Tr}\left(\mathbf{D}^{-1}\left(x_{L}\right) \mathbf{D}\left(x_{0}\right)\right)
$$

where for brevity we have defined

$$
x_{0}=\sqrt{\frac{E_{g 0}^{2} L}{\hbar v \Delta E_{g}}}, \quad x_{L}=\sqrt{\frac{E_{g L}^{2} L}{\hbar v \Delta E_{g}}} .
$$

Finally, using the relationship between parabolic cylinder functions and the confluent hypergeometric functions $M(\alpha, \beta ; t)$ [6], it is straightforward although somewhat tedious to demonstrate that the dispersion relation can be expressed as

$$
\begin{aligned}
& \cos k L=\frac{e^{-\left(x_{0}^{2}+x_{L}^{2}\right) / 4}}{2}\left\{M\left(-\frac{\eta}{2}, \frac{1}{2} ; \frac{x_{0}^{2}}{2}\right) M\left(\frac{1-\eta}{2}, \frac{1}{2} ; \frac{x_{L}^{2}}{2}\right)+M\left(-\frac{\eta}{2}, \frac{1}{2} ; \frac{x_{L}^{2}}{2}\right) M\left(\frac{1-\eta}{2}, \frac{1}{2} ; \frac{x_{0}^{2}}{2}\right)\right. \\
& \left.+\eta x_{0} x_{L}\left[M\left(\frac{1-\eta}{2}, \frac{3}{2} ; \frac{x_{0}^{2}}{2}\right) M\left(1-\frac{\eta}{2}, \frac{3}{2} ; \frac{x_{L}^{2}}{2}\right)+M\left(\frac{1-\eta}{2}, \frac{3}{2} ; \frac{x_{L}^{2}}{2}\right) M\left(1-\frac{\eta}{2}, \frac{3}{2} ; \frac{x_{0}^{2}}{2}\right)\right]\right\} .
\end{aligned}
$$

Whenever the absolute value of the right-hand-side of this equation is less than unity, a real value of $k$ is found and hence the dispersion relation inside allowed minibands is obtained. Conversely, if the absolute value is larger than unity, the energy corresponds to a minigap of the superlattice. 
As a specific example we have considered graded structures with $E_{g 0}=0.18 \mathrm{eV}$ (corresponding to $\mathrm{InSb}$ ), $E_{g L}=0.27 \mathrm{eV}, \hbar v=0.70 \mathrm{eV} \mathrm{nm}$ and superlattice periods $L$ ranging from $5 \mathrm{~nm}$ up to $40 \mathrm{~nm}$. Results of the allowed minibands and minigaps as a function of the lattice period are shown in Fig. 1. Note that allowed minibands shrink on increasing superlattice period due to the reduction of the overlap of neighbouring cells.

In conclusion, we have described theoretically the miniband structure in graded-gap superlattices within a two-band semiconductor model, that is, we have taking into account the coupling between the conduction- and valence-bands in the host semiconductor. Assuming that the gap increases linearly with position, we are able to solve exactly the $2 \times 2$ Dirac-like equation of the model. The dispersion relation inside allowed minibands may be expressed in a closed form in terms of the confluent hypergeometric functions. 


\section{REFERENCES}

[1] F. Capasso, S. Lurti, W. T. Tsang, C. G. Bethea, and B. F. Levine, Phys. Rev. Lett. 51, 2318 (1983).

[2] J. A. Brum, P. Voisin, and G. Bastard, Phys. Rev. B 33, 1063 (1986).

[3] G. Bastard, Phys. Rev. B 24, 5693 (1981). Rev. B 40, 6420 (1989).

[4] F. Domínguez-Adame and M. A. González, Europhys. Lett. 13, 193 (1990).

[5] F. Domínguez-Adame and B. Méndez, Il Nuovo Cim. B 107, 489 (1992).

[6] M. Abramowitz and I. Stegun (ed), Handbook of Mathematical functions (Dover, New York, 1972). 


\section{FIGURES}

FIG. 1. Miniband structure of a graded-gap superlattice within the two-band model, as a

function of the superlattice period. Energies are measured from the bottom of the conduction band at $z=0$. Shaded areas correspond to allowed minibands in the superlattice. 